\title{
SKIN FRICTION ESTIMATION IN A STRONG DECELERATING FLOW
}

\author{
Artur Dróżdż, Witold Elsner, DaWid Sikorski \\ Czestochowa University of Technology, Częstochowa, Poland \\ e-mail:welsner@imc.pcz.czest.pl
}

The paper presents the analysis of the turbulent boundary layer developed on a flat plate subjected to an Adverse Pressure Gradient (APG) and approaching separation. The aim of the study is to examine the effects of pressure gradient on a non-equilibrium boundary layer while indicating local areas of the equilibrium flow. The emphasis is on the analysis of mean flow velocity and the estimation of skin friction. It is known that accurate measurements of skin friction were considered as a difficult and demanding task despite of various measuring techniques available. A great challenge is especially the measurement of a strong decelerated turbulent boundary layer because of low shear stress and possible large measuring errors. To date, the oil film or oil drop interferometry technique, because of its high accuracy, has become a basis of turbulent-boundary-layer research. In our research, this technique has been used as a reference method for comparing with the traditional Clauser chart method, which generally is considered as not suitably for non-canonical flows. In the paper, however, a correction of the method is proposed, which allows one to increase its range of applicability. This corrected Clauser chart method (CCCM) involves only one iteration while other proposed in the literature methods employ a twofold iterative procedure. The comparison of the methods for the non-canonical turbulent boundary layer, i.e. adverse pressure gradient with a strong flow history effect has been presented. It has been shown that CCCM can be successfully used for small and medium pressure gradients, where the Clauser-Rotta pressure gradient parameter $\beta$ does not exceed level close to 11.

Keywords: turbulent boundary layer, separation, oil-film interferometry, Clauser chart

\section{Introduction}

The measurement of skin friction is presently recognized as a critical element of aerodynamic testing. It gives rise to crucially important flow phenomena such as viscous drag on air and ground vehicles, on wind turbine blades, and losses in internal flows. It provides critical information necessary for computational simulations and serves as a sensitive quantity for use in flow-control applications.

Until now, accurate measurements of skin friction were considered as a difficult and demanding task as it requires precise measurements of mean velocity in the viscous sublayer, where the determination of the velocity gradient is necessary (Hutchins and Choi, 2002). Most commonly used for this purpose is a measuring method relying on hot-wire technique. Despite theoretical basis, the accuracy of this method is strongly dependent on resolution and quality of measurements very close to the wall (Dixit and Ranesh, 2009). According to Castillo and Johansson (2002), this method works satisfactorily only for low Reynolds number flows. Concerning adverse pressure gradient (APG) flows, where the low velocity close the wall occurs, the results from hotwire measurement are biased by heat transfer to the wall (Ikeya et al., 2017). An indirect method based on the measurement of velocity profile, the so called Clauser plot or Clauser chart method (Clauser, 1956) (herein CCM), do not require precise measurements in the viscous sublayer as it is based on the logarithmic overlapping layer universality assumption. In its original form it 
is however, restricted only to canonical flows. There are some modifications of the method for equilibrium or near-equilibrium pressure gradient flows, which are based on the non-universal or pressure-gradient-dependent log law in the inner scaling (Dixit and Ranesh, 2009), however their applications are limited as they provide different parameters in to the log-law function for pressure gradient changes without analyzing flow history effects (Bobke et al., 2017; Dróżdż and Elsner, 2017).

On the other hand, the method considered to be the most accurate is the oil film or oil drop interferometry technique which is based on the work of Tanner and Blows (1976). This technique uses the dependency between the thinning of an oil film deposited on the surface exposed to the flow and the local shear stress. According to (Segalini et al., 2015), if carefully implemented, this method yields the true time averaged skin friction within the $\pm 1 \%$ of measurement accuracy.

The paper presents results of skin friction measurements using the two above methods applied for a strong decelerated turbulent boundary layer developed on the flat plate being at the verge of separation.

The goal of the study is to verify applicability of this method for a very demanding case, where a low shear stress is present and where its estimation may be associated with large measuring errors. In the paper, the comparison of the measurements accuracy with the proposed correction to the Clauser chart method for a strong pressure gradient flow with the flow history effect, is presented.

\section{Methodology and instrumentation}

An open-circuit wind tunnel located at Czestochowa University of Technology has been used for this experiment. The facility consists of a blower, settling chamber, and a long rectangular channel with length of $5.035 \mathrm{~m}$ located upstream the test section. As a result, the turbulent boundary layer develops on a long plate what allows it to reach boundary layer thickness up to $90 \mathrm{~mm}$ at the inlet to the test section. The inlet rectangular channel has two pairs of suction gaps aimed, at maintaining overpressure, to control the two-dimensionality of the flow by minimizing the boundary layers on the side walls. Triangular corner inserts are used in the whole inlet channel to reduce the effect of secondary vortices developing along the rectangular channel. A slight inclination of the upper wall helps maintaining zero pressure gradient $\left(d P_{\infty} / d x \approx\right.$ 0 , where $P_{\infty}$ is external static pressure and $x$ is the streamwise direction) conditions at the inlet.

The specially design diffuser shape test section with length of $1.835 \mathrm{~m}$ (see Fig. 1) is equipped with a perforated movable upper wall. Wall perforation of $10.1 \%$ is adopted, characterized by $0.5 \mathrm{~mm}$ circular holes. Modification of the shape and position of the upper wall and the suction flux enables generation of a wide range of pressure gradient conditions, while the zero pressure gradient conditions are maintained at the inlet channel. With specific pressure conditions, it is possible to obtain, on the bottom wall, a turbulent boundary layer which is at the verge of separation. Full separation on the lower flat plate does not occur even for the suction case. After that point, due to the cessation of suction, the flow returns to the attached state.

The velocity measurements have been performed with a single hot-wire anemometry probe of diameter $d=3 \mu \mathrm{m}$ and length $l=0.4 \mathrm{~mm}$ (modified Dantec Dynamics 55P31). The probe was a combined with hot-wire anemometry CCC developed by the Polish Academy of Science in Krakow.

The hot-wire bridge was connected to a 16 bit A/D converter. The acquisition was maintained at the frequency of $25 \mathrm{kHz}$, with minimum $30 \mathrm{~s}$ sampling records. The ambient conditions were carefully controlled during the measurements. During single profile measurements, the scatter of ambient temperature did not exceed $\pm 0.2^{\circ}$. If the measured temperature was different from 
(a)

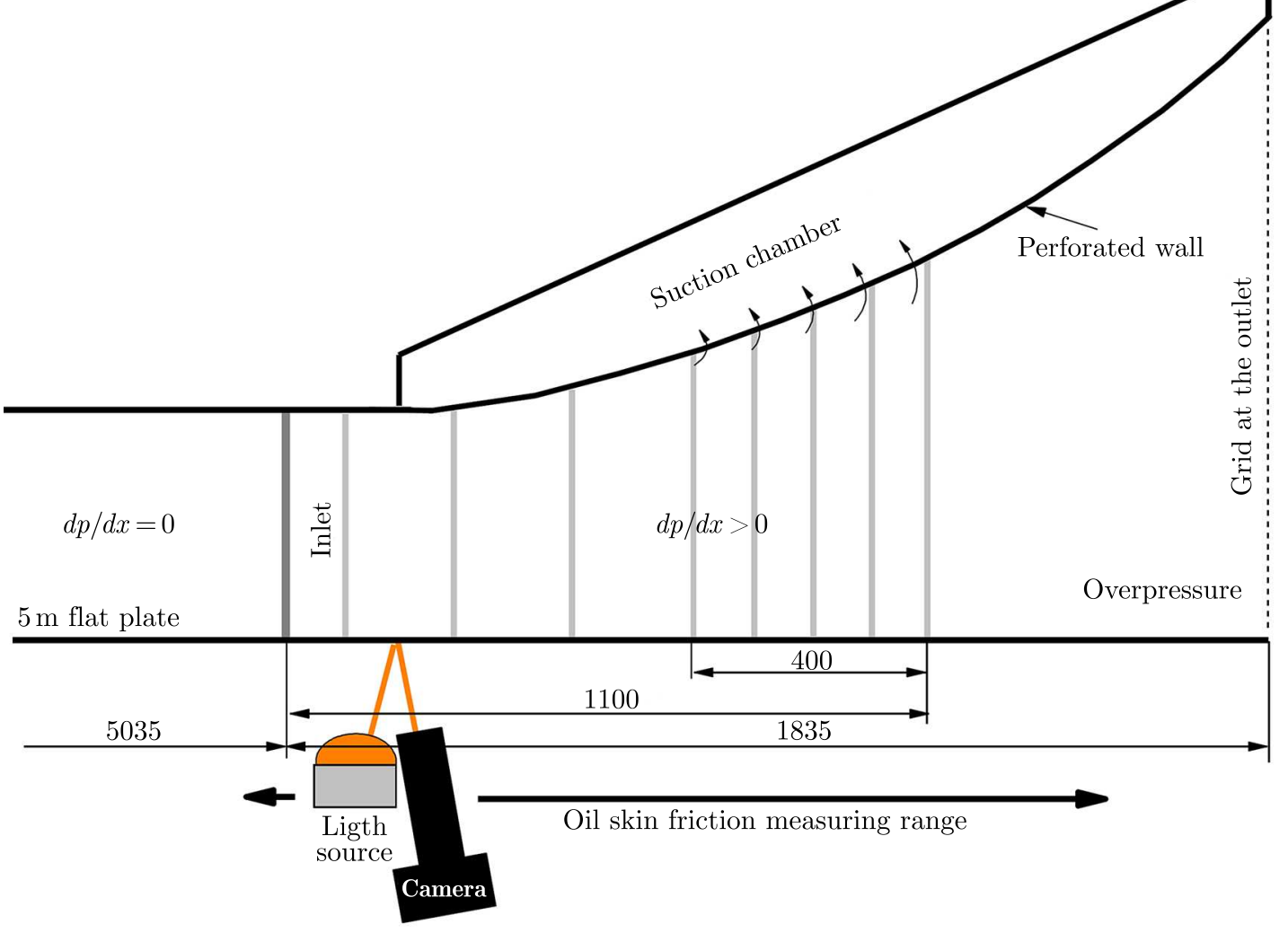

(b)

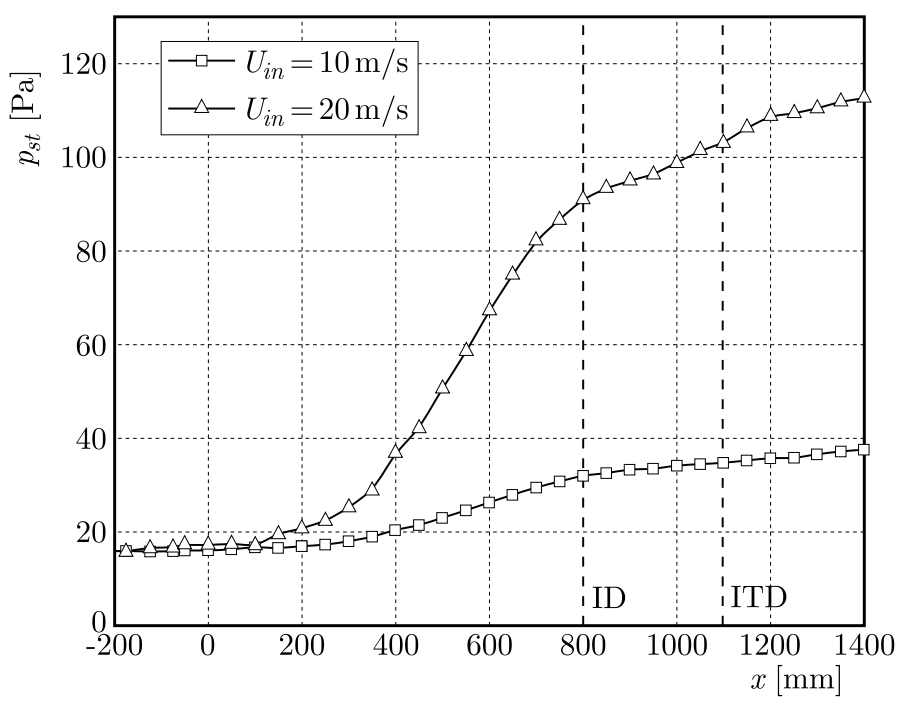

Fig. 1. Test section geometry (a) and pressure distribution (b)

the calibration temperature, the temperature correction of CTA voltage was used by Jorgensen (2002). Free-stream velocity was simultaneously monitored by means of the Prandtl tube.

The velocity measurements were performed for two inlet velocities $U_{i n}=10$ and $20 \mathrm{~m} / \mathrm{s}$, which corresponded to the Reynolds number based on momentum loss thickness 6300 and 10150. Basic inlet parameters are summarized in Table 1, where $T u$ is turbulence intensity, $U_{\text {in }}$ mean velocity outside the turbulent boundary layer, $u_{\tau}$ friction velocity $\left(u_{\tau}=\sqrt{\tau_{w} / \rho}\right), \theta$ momentum loss thickness and $\operatorname{Re}_{\theta}=U_{i n} \theta / \nu$ is the Reynolds number, where $\nu$ is the kinematic viscosity. The pressure distribution imposed by the upper wall and the active suction is presented in Fig. 1b. Further details of the experiment can be found in (Dróżdż and Elsner, 2017). 
Table 1. Inlet conditions of ZPG turbulent boundary layer

\begin{tabular}{|c|c|c|c|c|c|}
\hline & $T u[\%]$ & $U_{\text {in }}[\mathrm{m} / \mathrm{s}]$ & $u_{\tau}[\mathrm{m} / \mathrm{s}]$ & $\theta[\mathrm{mm}]$ & $\operatorname{Re}_{\theta}[-]$ \\
\hline \hline 1 & $0.7 \%$ & 10 & 0.37 & 8.26 & 6300 \\
\hline 2 & $0.7 \%$ & 20 & 0.72 & 10.3 & 10150 \\
\hline
\end{tabular}

\section{Methodology of direct skin friction estimation}

The direct method of skin friction estimation is based on the fringe skin friction (FSF) technique introduced by Tanner and Blows (1976), who relate the evolution of oil droplet thickness to the skin friction $\tau_{w}$. The method is based on the assumption of constant shear stress for a given measuring point already used in experimental investigations (Dróżdż et al., 2008; Pailhas et al., 2009). Under the action of the flow shearing force the oil film is getting thinner leading, under lighting of the monochromatic lamp, to a series of interferometric fringes. These fringes are produced as a result of interference of the light reflected from the surface and from the air-oil interface. Distance between consecutive fringes is strictly related to the oil layer thickness and consequently to skin friction.

For the purpose of skin friction measurement, the optical equipment has been installed in the wind tunnel under the plate (see Fig. 1a). It consisted of commercial camera equipped with Macro lens and SOX Whitecroft Lighting sodium lamp emitting the monochromatic light of wavelength $\lambda=0.5893 \mu \mathrm{m}$ used to illuminate the oil droplet. For the measurements, the OM50 silicone oil with viscosity of about 0.048 Pas was used. The accuracy of wall shear data was in the range of $1 \%$, which corresponded to $0.5 \%$ accuracy in friction velocity for the inlet $u_{\tau}$. The oil viscosity is the most important parameter that has to be precisely controlled that is why temperature of the flow, which is also the temperature of the flat plate, was carefully measured using a temperature sensor. In the course of a single profile measurement the scatter of ambient temperature at the end of the test section did not exceed $\pm 0.2^{\circ}$ and the temperature difference between the flowing air and the wall was also below $0.1^{\circ} \mathrm{C}$.

In the central part of the flat plate, an optical glass delivered by Schott Company has been mounted. This glass, used for radiological shields due to high lead contents, is characterized by a very smooth surface, which is necessary to get smallest distortion of the fringe pattern. The camera has been controlled by a computer program, which triggered the shutter from every $10 \mathrm{~s}$ up to $30 \mathrm{~s}$ dependending on the flow speed. Pictures have been recorded as monochromatic and processing of the images obtained from experiments has been performed in Matlab Image Processing Toolbox. Quality of images has been improved by image contrast adjustment and histogram equalization procedures, which have been adopted from Matlab library (Dróżdż et al., 2008).

The direct estimation of skin friction has been obtained from a series of pictures (minimum 25) of fringe patterns forming on the oil droplet thinning under the influence of the flow shear force. An exemplary image of the thinning droplet is presented in Fig. 2. Extracted pixels of varying brightness from the single line from oil symmetry plane are used in order to estimate the fringe spacing. Having the estimated fringe spacing from all taken pictures, the distribution of the period versus time is obtained (Fig. 3). The approximation of the distribution with least squares algorithm allows one to determine the slope of the curve $a$.

The shear stresses $\tau_{w}[\mathrm{~Pa}]$ is calculated from the following equation

$$
\tau_{w}=\frac{2 n_{o} \mu_{o} a}{c \lambda} \cos \theta_{o}
$$

where $n_{o}=1.4$ is the index of refraction for the oil, $\mu_{o}$ - dynamic viscosity of the oil, $c$ - calibration coefficient [pixels/mm], $a$ - slope of the fringe spacing versus time [pixels/s] 


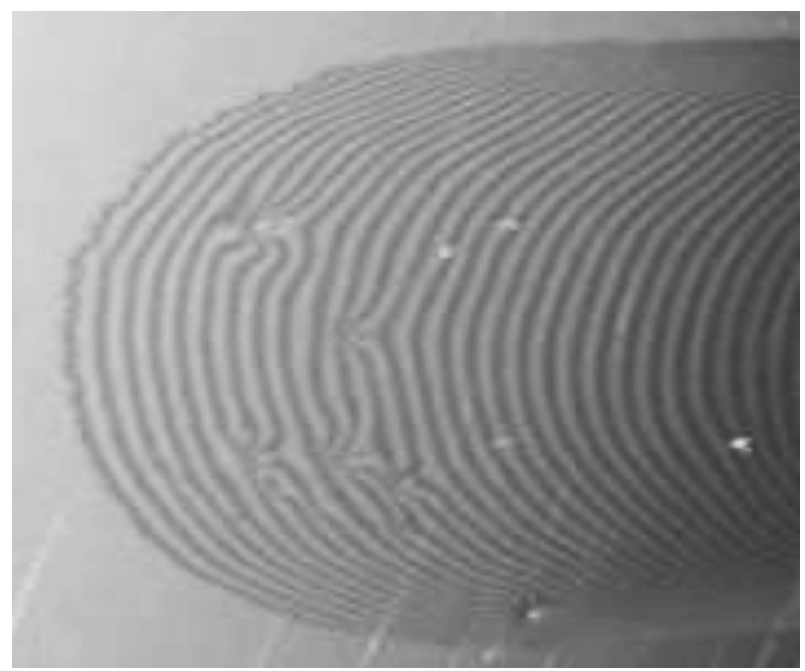

Fig. 2. Oil droplet with the interferometry pattern

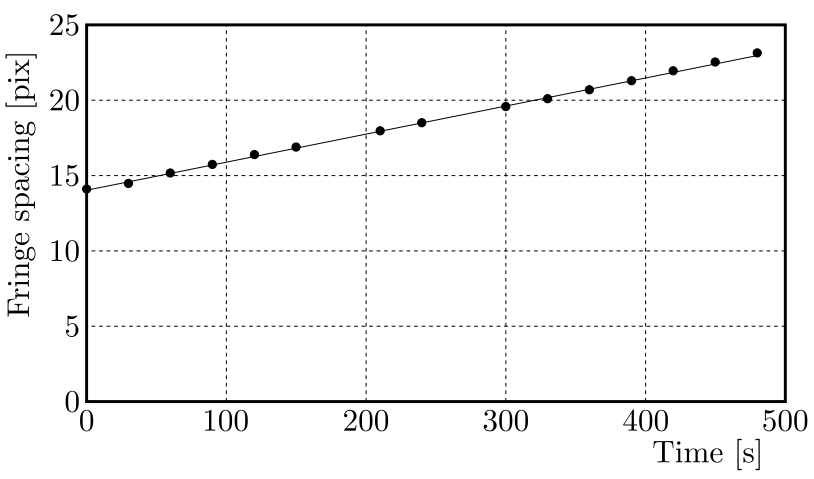

Fig. 3. Fringe spacing versus time

and $\theta_{o}$ is the angle between the incident light and the direction normal to the plate in the oil environment. In comparison with Pailhas et al. (2009), the present shear stresses formula (3.1) includes the influence of the view angle in the oil environment $\theta_{o}$. It is important to know that the viewing angle can lead to overestimation of the skin friction error equal to $0.7 \%$ for the angle $\theta=10^{\circ}$ in the air environment.

\section{Methodology of corrected Clauser chart method (CCCM)}

The indirect estimation of skin friction can be realized using the Clauser plot method (Clauser, 1956). It is based on the assumption of universality of the logarithmic overlap region for zero pressure gradient flows defined as

$$
U^{+}=\frac{1}{\kappa} \ln \left(y^{+}\right)+B
$$

where the von Karman constant $\kappa$ and constant $B$ are independent of the Reynolds number. It is common knowledge that this method is considered not very accurate because, among others, there is no value of constant $\kappa$ that would be widely accepted (Zanoun et al., 2003). As often reported in the literature, e.g. (Kendall and Koochesfahani, 2007) the Clauser plot method produces artificially high friction velocities, especially at the low range of Reynolds numbers. For example, for Reynolds numbers in the range of $\operatorname{Re}_{\theta}=1000-10000$ (Blackwelder and Haritonidis, 1983), the friction velocity was reported to have an error varying between $8 \%$ and $20 \%$ while at 
very high Reynolds numbers, the larger extent of a log-linear region improved the accuracy of the Clauser method provided that the value of $\kappa$ was correctly adopted. According to Nagib et al. (2004) for a zero pressure gradient turbulent boundary layer the most of the data are very well represented using the $\log$-law with $\kappa=0.38, B=4.1$, and the same constants are adopted in the current investigations.

Originally, Clauser chart method involved fitting of the velocity profile with log-line in the overlap layer by changing the value of friction velocity $u_{\tau}$. In practice, there is also a need to select beginning and end of the region where the logarithmic velocity profile occurs. It introduces user subjectivity, which can result in substantial errors, especially for low Reynolds numbers and for TBL with a strong adverse pressure gradient, where the log region in the overlap layer is hardly observed. For the APG flow the region occupied by the log-law is progressively reduced and the mean velocity profiles will deviate from the logarithmic line resulting in a change of $\kappa$ and $B$ constants. In the absence of direct measurements of the shear stress, the attempt to preserve constants valid for the ZPG flow, the logarithmic region shifts the profile to the wall. This causes underestimation of shear stress values as shown at Fig. 4.

(a)

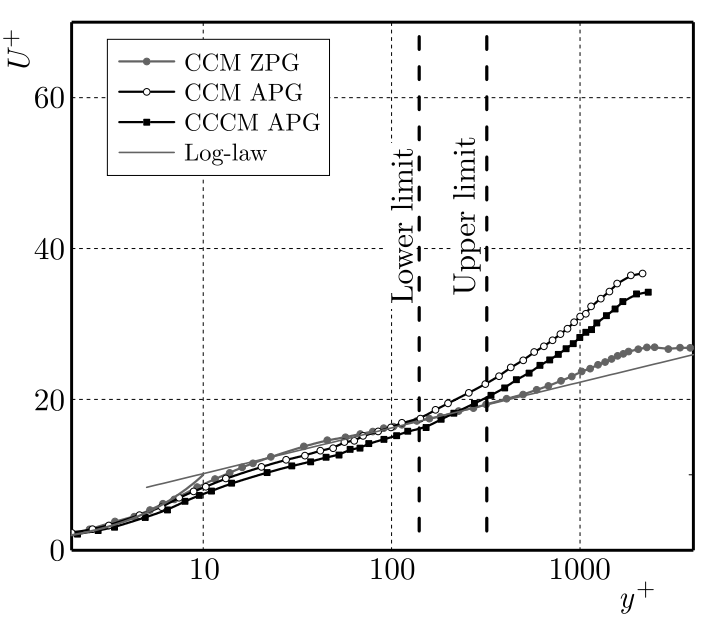

(b)

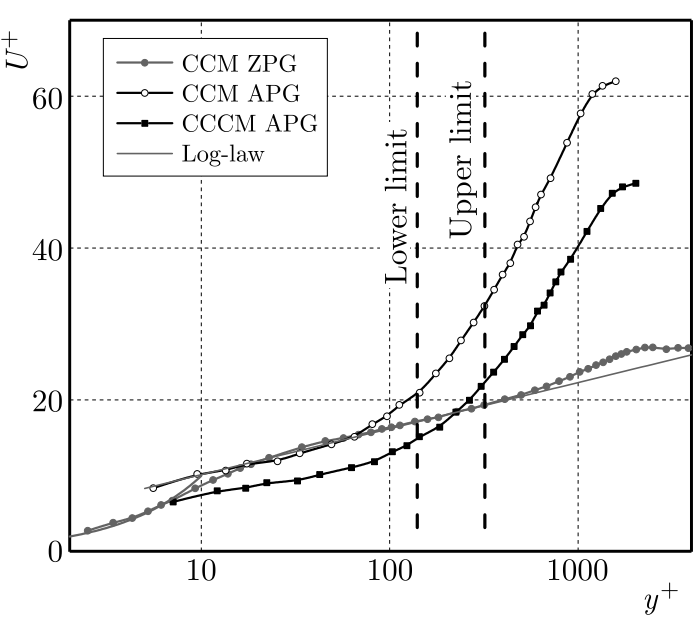

Fig. 4. Comparison of inner scaled mean velocity profiles for $U_{i n}=10 \mathrm{~m} / \mathrm{s}$ estimated by the original and corrected Clauser plot method for $x=700 \mathrm{~mm}$ and $x=900 \mathrm{~mm}$

The aim of this study is to propose a very simple extension of the conventional Clauser chart method based on the assumption that the preceding ZPG turbulent boundary layers are correctly defined in viscous units and so that the lower and upper limits of the overlapping logarithmic layer for the analysed Reynolds number are known. It is generally accepted that the near-wall flow is vulnerable to changes in the pressure gradient, preserving however memory of the upstream flow features. As it was mentioned above, the basic problem for this type of flow is the reduction of the logarithmic zone with an increase in $\beta$ while for the boundary layer approaching separation the mean velocity profile does not even reveal a log region at all. Our concept stems from Mathis et al. (2009) observation that for ZPG TBL the centre of large scale structures coincides well with the geometrical centre of the logarithmic region. It could be assumed, therefore, that large scales have an important effect on the shear stress at the wall. However, for APG flow, the region occupied by large-scales is extended above the log region that is why the range of occurrence of the large scale motions, which is the same as the overlap log layer properly defined for ZPG (Dróżdż and Elsner, 2017), should be used rather than the log region. As it is suggested in a number of papers e.g. (Vinuesa and Nagib, 2015), the lower boundary of overlapping log layer for ZPG TBL is invariant with the Reynolds number $\left(y^{+} \approx 150\right)$, while the upper boundary can be calculated at a value of $y^{+}=0.15 \delta^{+}$(as the inner range of the near wall region is $y / \delta=0.15$ ) (Mathis et al., 2009), where $\delta^{+}=\delta u_{\tau} / \vartheta$ 
is the zero pressure gradient turbulent boundary layer thickness $\delta$ presented in viscous units. The above boundaries can be estimated in few iterations using the Clauser chart for the zero pressure gradient case and should be kept constant for the consecutive profiles in APG region, even if there is no logarithmic profile. For flows with $\delta^{+}<1000$, the log region does not exist because the upper and lower boundaries are equal. In this case, the boundaries may be modified slightly by \pm 50 viscous units (to obtain range 100-200) to cover at least a few measuring points. Assuming the invariable location of the logarithmic profile in the overlap region, already defined for ZPG where the large scales centers reside, the location of the experimental APG profile is modified using the least-square method such that the R-squared value of the fit, with the logarithmic curve within the above defined overlap layer limits, reaches a maximum (keeping constants equal $\kappa=0.38$ and $B=4.1$ ).

The concept is presented for a lower Reynolds number case in Fig. 4, where two selected mean velocity profiles for two locations $x=700 \mathrm{~mm}$ (Fig. 4a) and $x=900 \mathrm{~mm}$ (Fig. 4b) are drawn in inner scaling. Each figure contains the mean ZPG profile (grey dots), the profile plotted according to the original Clauser method (open circles) and the profile plotted according to the corrected Clauser method (dots) as well as the log law line for ZPG flow. In the figures, the boundaries of the defined overlap region are drawn as vertical dashed lines. The first location corresponds to the pressure gradient parameter $\beta=11$ while the other location corresponds to the incipient detachment (ID) point according to the definition of Simpson (1989), where the pressure gradient parameter $\beta$ equals to 28 .

In both cases, the mean velocity profile drawn according to the conventional Clauser chart method (CCM) is fitted to the log-law but in the region much closer to the wall. Such a fit of the mean profile causes undervaluation of friction velocity $u_{\tau}$. The CCCM allows one to correct the friction velocity by about $6.7 \%$ (from 0.235 to 0.252 ) for $\beta=11$, which is consistent with the results obtained by the oil-film interferometry method and by about $21.7 \%$ (from 0.13 to 0.166 ) for $\beta=28$. There is of course a question about the range of applicability of this methodology and it will be presented in the next Section of the paper.

\section{Flow conditions}

The diverging measuring section enables generation of a predetermined adverse pressure gradient. The flow conditions in the section can be best described by the pressure coefficient

$$
C_{p}=1-\left(\frac{U_{\infty}}{U_{\infty 0}}\right)^{2}
$$

Here $U_{\infty}$ is the local and $U_{\infty 0}$ is the inlet freestream velocity determined for $x=0 \mathrm{~mm}$. The distributions of $C_{p}$ are presented in Fig. 5a while Fig. 5b presents the Clauser-Rotta pressure gradient parameter $\beta$. The rise of $\beta$ to 90 for the streamwise distance $x=1100 \mathrm{~mm}$ generates conditions that increase the susceptibility of the boundary layer to detachment. It should be also noted that, irrespective of two different Reynolds numbers, both $C_{p}$ and $\beta$ distributions match well. This confirms that from the point of view of the pressure gradient it is possible to obtain similar external conditions.

Prior to flow analysis in the APG area, verification of the inlet conditions is necessary. The first travers i.e. for $x=0 \mathrm{~mm}$ is located at the region of zero pressure gradient, so it is easy to check the measured skin friction data against explicit relation of Coles-Fernholz $C_{f}=2\left[(1 / \kappa) \ln \left(\operatorname{Re}_{\theta}\right)+B\right]^{-2}$, which was modified by Nagib et al. (2004) (modification of $\kappa$ and $B$ is valid for the zero pressure gradient boundary layer). The measured, using oil film interferometry, values of skin friction are used to calculate friction coefficients applying $C_{f}=2 \tau_{w} /\left(\rho U_{\infty}^{2}\right)$ formula. The data presented in Fig. 6 confirm the agreement of oil film interferometry readings with the theoretical line in function of the Reynolds number. 
(a)

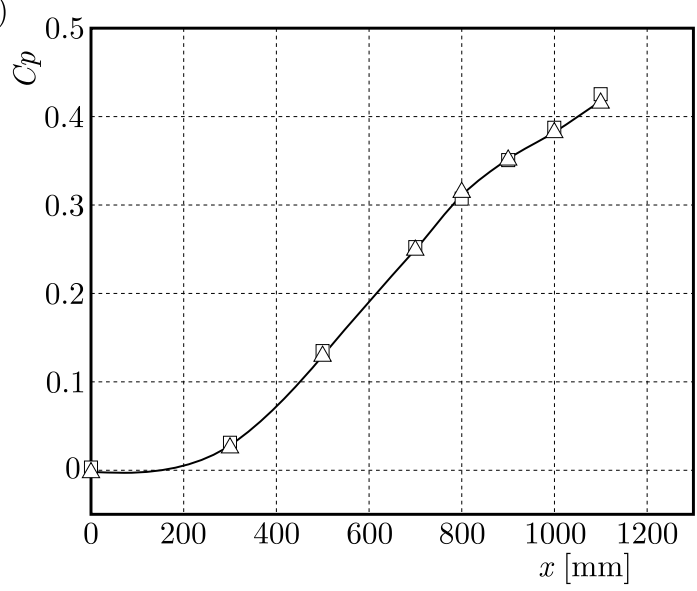

(b)

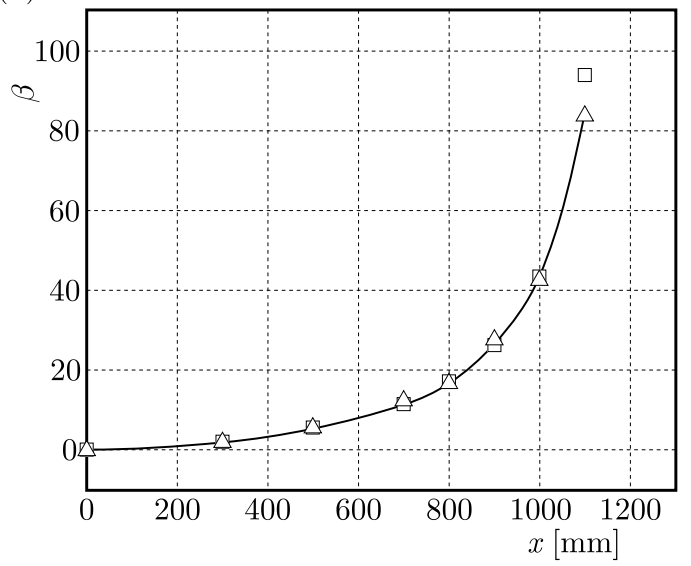

Fig. 5. Distributions of the flow parameters: pressure gradient parameter $C_{p}$ (a) and Clauser-Rotta pressure gradient parameter $\beta$ (b)

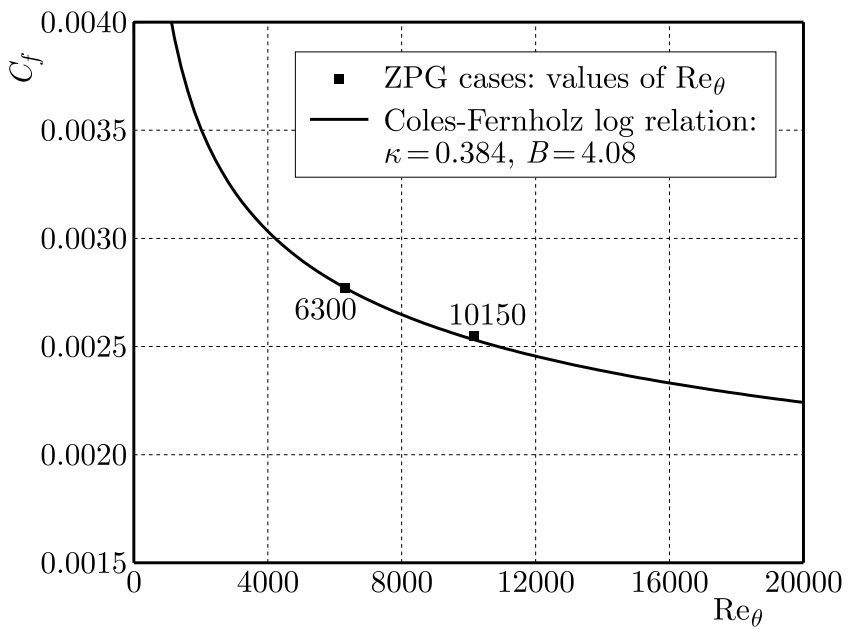

Fig. 6. Friction coefficient relation with the Reynolds number measured at the inlet plane

\section{Discussion of the results}

Having verified inlet conditions the indirect estimation of skin friction is performed using the Clauser chart method (Clauser, 1956) for consecutive APG traverses up to the incipient of separation. Figure 7 shows the distribution of skin friction along the plate using the CCM (squares) as well as CCCM (circles). Close to the separation, the values obtained by both methods are compared with the reference data obtained by oil-film interferometry (open triangles). For these latter data, the level of measurement uncertainty is shown. Since the measurement uncertainty in absolute terms is estimated to be at the level $0.006 \mathrm{~Pa}$ (which is $1 \%$ of the ZPG value), the relative value, shown by the uncertainty bars, is almost four times higher for $10 \mathrm{~m} / \mathrm{s}$ (Fig. 7a) than for $20 \mathrm{~m} / \mathrm{s}$ (Fig. $7 \mathrm{~b}$ ).

It is clear from Fig. 7a that for lower Reynolds numbers both indirect methods provide consistent results for the distance $x$ up to $500 \mathrm{~mm}$. However, further downstream, where the Clauser-Rotta pressure gradient parameter $\beta \gtrsim 5.0$, the consistency is lost. The first oil fringe measurement was performed for the $x=700 \mathrm{~mm}$ and it was the same location where the difference between CCM and CCCM estimations did not agree. Results of the CCCM agree well with oil - fringe measurements and fall within the range of its measurement uncertainty. Further downstream for both Clauser based methods, a decrease of skin friction of about the same order 

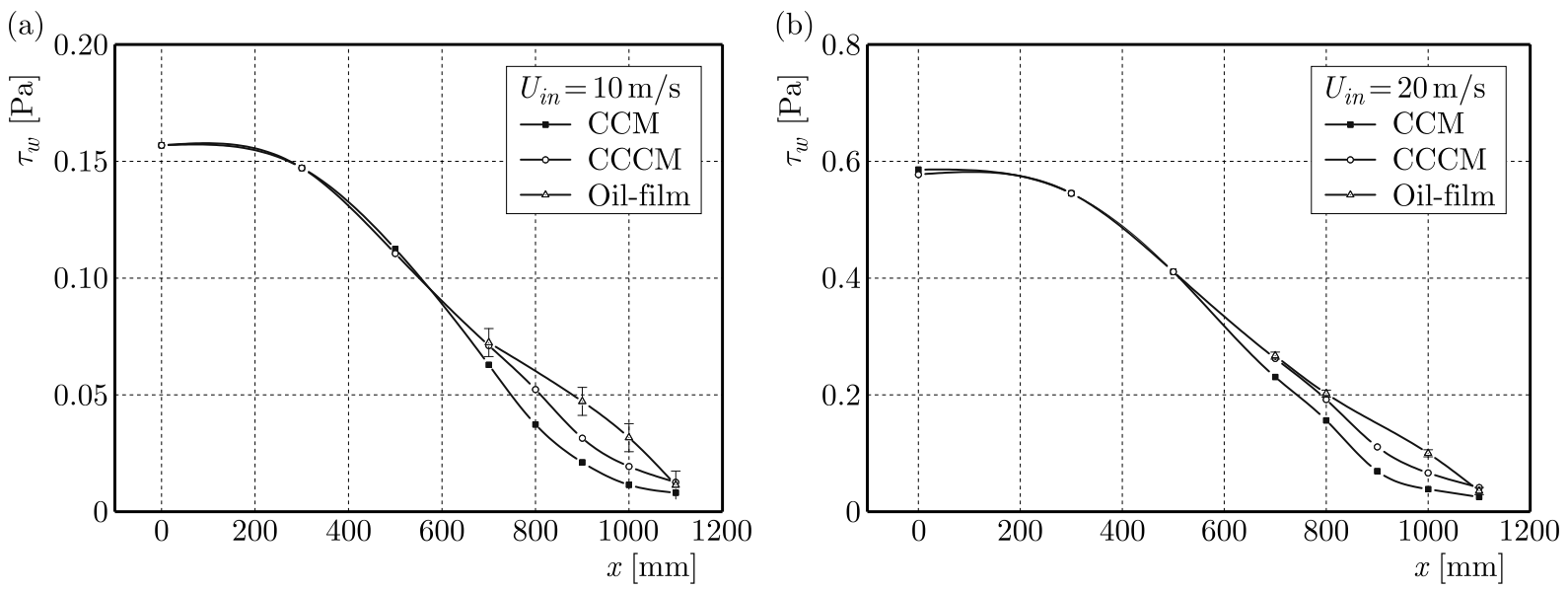

Fig. 7. Comparison of skin friction distributions of the Clauser chart and with the proposed correction verified with oil skin friction (a) $10 \mathrm{~m} / \mathrm{s}$ and (b) $20 \mathrm{~m} / \mathrm{s}$

but with lower values for the original Clauser chart is observed. A slightly different in character is the trend measured for the reference technique. Surprisingly, at the ITD point $(x=1100)$ all three estimations are pretty much the same. For higher Reynolds numbers (Fig. 7b), the stress values are much higher, but the tendency in $\tau_{w}$ changes is maintained. In the latter case, attention shall be paid to the comparability of the CCCM estimation with oil measurements even for pressure parameter $\beta$ close to $17(x=800 \mathrm{~mm})$. All measurement data are collected in Table 2. The values which are comparable with OFI method are marked in bold.

Table 2. Comparison of skin friction values

\begin{tabular}{|c|c|c|c|c|c|c|c|}
\hline \multirow{2}{*}{$\begin{array}{c}x \\
{[\mathrm{~mm}]}\end{array}$} & \multirow{3}{*}{$\begin{array}{c}\beta \\
{[-]}\end{array}$} & $\begin{array}{c}\text { CCM } \\
{[\mathrm{Pa}]}\end{array}$ & $\begin{array}{c}\text { CCCM } \\
{[\mathrm{Pa}]}\end{array}$ & $\begin{array}{c}\text { OFI } \\
{[\mathrm{Pa}]}\end{array}$ & $\begin{array}{c}\mathrm{CCM} \\
{[\mathrm{Pa}]}\end{array}$ & $\begin{array}{c}\text { CCCM } \\
{[\mathrm{Pa}]}\end{array}$ & $\begin{array}{c}\text { OFI } \\
{[\mathrm{Pa}]}\end{array}$ \\
\hline \hline 700 & 11 & 0.063 & $\mathbf{0 . 0 7 1}$ & $\mathbf{0 . 0 7 2}$ & 0.231 & $\mathbf{0 . 2 6 2}$ & $\mathbf{0 . 2 6 7}$ \\
\hline 800 & 17 & 0.037 & 0.051 & - & 0.156 & 0.192 & 0.202 \\
\hline 900 & 28 & 0.021 & 0.029 & 0.047 & 0.069 & 0.111 & - \\
\hline 1000 & 43 & 0.011 & 0.019 & 0.032 & 0.039 & 0.066 & 0.100 \\
\hline 1100 & 85 & $\mathbf{0 . 0 0 8}$ & $\mathbf{0 . 0 1 3}$ & $\mathbf{0 . 0 1 1}$ & 0.025 & $\mathbf{0 . 0 4 1}$ & $\mathbf{0 . 0 3 5}$ \\
\hline
\end{tabular}

As can be seen at the beginning of detachment $(x=800-1000 \mathrm{~mm})$, the Clauser chart method significantly lowers the skin friction. Lack of near wall flow similarity with canonical flow and a departure of the mean velocity profile from the log-line may be due to various reasons. According to Knopp et al. (2013), this could be attributed to a sudden change of the mean velocity in the streamwise direction. The explanation of this phenomenon can also be carried out based on the analysis of physics of the processes occurring near the wall of the comsidered flow as it was shown in (Dróżdż and Elsner, 2017). It is known that the adverse pressure gradient enhances the large- and small-scale interaction which leads to the rise of small scale convection velocity near the wall. This mechanism is responsible for increasing the momentum near the wall, which leads to the rise of the wall skin friction. The Clauser plot method does not take account of this process because it is based on the assumption that the mean velocity is universal in the whole inner region including the log layer, which is not valid for APG. Since CCCM, to some extent, takes into account the influence of large scale motion that is why its estimations are closer to reality. Close to separation (ITD point), due to the drop of energy of the small scales (convection velocity is no longer efficient in increasing the momentum), this effect is weaker, which may be the reason for more consistent results obtained by all methods. 
Available literature data (Madad et al., 2010) suggest that the Clauser chart method is applicable only for zero and weak adverse pressure gradient flows. They showed that beyond $\beta=2.0$ the difference in the reference to the oil interferometry method becomes significant (approximately $10 \%$ difference of $C_{f}$ ). However, the proposed modification extends the upper limit of usable range of the Clauser chart method from $\beta \approx 2.0$ (Monty et al., 2011) up to $\beta \approx 11.0$. On the other hand, for low friction velocity and close to separation, the application of any method is problematical because of large uncertainty level.

It is well known that the impact of large-small scale interaction on the near wall region increases with the Reynolds number. As it was shown in (Dróżdż and Elsner, 2017), the difference between velocity profiles for two considered Reynolds numbers was not significant even close the separation. However, the Reynolds stress profiles and spectral analysis shows the increase of small scale energy close the wall with the Reynolds number due to the process of modulation of near wall small scales by large scales from the outer zone. An increase of Reynolds stress indicates an increase of the strain rate $(d U / d y)$ close the wall, therefore it can be expected that it will also be noticeable on skin friction. In order to show the effect of the Reynolds number on the turbulent boundary layer for the same distribution of $C_{p}$ in this area the results from Fig. 7 (only CCCM and OFI) are reduced by the inlet zero pressure gradient (ZPG) friction velocity $u_{\tau Z P G}$ and are shown in Fig. 8 . The oil fringe values are approximated by a linear function (dashed dot line for $10 \mathrm{~m} / \mathrm{s}$ and dashed line for $20 \mathrm{~m} / \mathrm{s}$ ). The uncertainty levels are also provided in relevant scaling. The results demonstrate that for the same pressure conditions, although differing in the number of Re, the distributions of reduced friction velocity do not reveal influence (in the uncertainty range). The reason for the lack of the Reynolds number effect may be due to a low Reynolds number difference. Further research should be carried out for a wider range of Reynolds numbers to prove the effect.

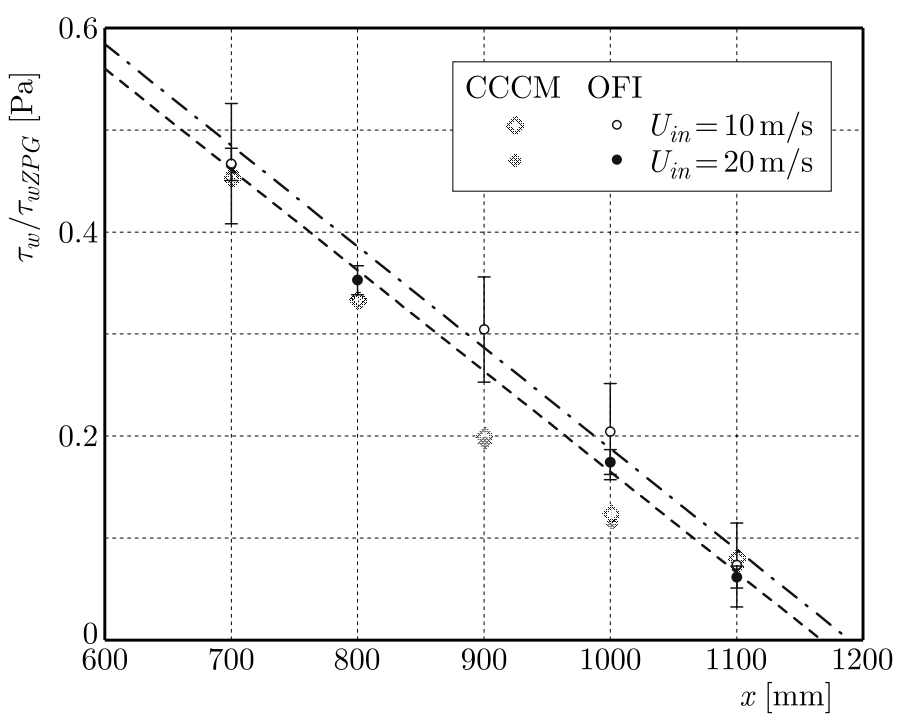

Fig. 8. Reduced skin friction distributions obtained with oil-film interferometry and CCCM. Reduced by its ZPG value

\section{Summary and conclusions}

The goal of the study is to verify applicability of the Clauser chart method and thin-oil film interferometry technique for a very demanding case. Difficulty of the analyzed problem does not rise only form a strong history effect of the flow, but also from a low shear stress, where their estimation may be associated with large measuring errors. The skin friction measurements in the turbulent boundary layer approaching separation are performed for two different Reynolds 
numbers with the same distribution of the pressure coefficient $C_{P}$. The results are compared with the corrected Clauser chart method (CCCM) which is introduced in the paper. This corrected Clauser chart method involves only one iteration, while the other methods, as the modified Clauser chart method (MCCM), employ a twofold iterative procedure (one iteration on $C_{f}$ and the other on pressure gradient coefficient). It is found that the skin friction distributions obtained by the CCCM method agree very well with the reference oil-film interferometry data (within the uncertainty range of oil-film interferometry) up to Incipient Detachment point. Further downstream, an underestimation of the CCCM in reference to the oil-film interferometry method is observed. An explanation of this effect is proposed, in which attention is paid to the importance of large-small scales interaction leading to deformation of the mean velocity profile near the wall which makes the Clauser plot method inapplicable for flows with strong pressure gradient. This effect is present up to Intermittent Transitory Detachment point, where due to increased fraction of reversed flow the drop of energy of the small scales is observed. Close to the separation $\left(\tau_{w}<0.3 \mathrm{~m} / \mathrm{s}\right)$, applicability of any method is problematic because of large uncertainty of any applied techniques. The effect of the Reynolds number for the same pressure gradient conditions is not observable for the analysed Reynolds number range.

\section{Acknowledgement}

The investigation was supported by National Science Centre under Grant No. DEC$-2012 / 07 / \mathrm{B} / \mathrm{ST} 8 / 03791$.

\section{References}

1. Blackwelder R.F., Haritonidis J.H., 1983, Scaling of the bursting frequency in turbulent boundary layers, Journal of Fluid Mechanics, 132, 1, 87

2. Bobke A., Vinuesa R., Örlü R., Schlatter P., 2017, History effects and near-equilibrium in adverse- pressure-gradient turbulent boundary layers adverse-pressure-gradient turbulent, Journal of Fluid Mechanics, 820 (April), 667-692

3. Castillo L., Johansson T.G., 2002, The effects of the upstream conditions on a low Reynolds number turbulent boundary layer with zero pressure gradient, Journal of Turbulence, 3, 31, 1-19

4. Clauser F.H., 1956, The turbulent boundary layer, Advances in Applied Mechanics, 4, 1-51

5. Dixit S.A., RAmesh O.N., 2009, Determination of skin friction in strong pressure-gradient equilibrium and near-equilibrium turbulent boundary layers, Experiments in Fluids, 47, 6, 1045-1058

6. DróżDż A., ElSNer W., 2017, An experimental study of turbulent boundary layers approaching separation, International Journal of Heat and Fluid Flow, 68C (April), 337-347

7. DróżDż A., Elsner W., Drobniak S., 2008, Application of oil-fringe interferometry for measurements of wall shear stress, Turbomachinery, 133, 103-110

8. Hutchins N., Choi K.S., 2002, Accurate measurements of local skin friction coefficient using hot-wire anemometry, Progress in Aerospace Sciences, 38, 4/5, 421-446

9. Ikeya Y., Örlü R., Fukagata K., Alfredsson P.H., 2017. Towards a theoretical model of heat transfer for hot-wire anemometry close to solid walls, International Journal of Heat and Fluid Flow, 68, 248-256, DOI: 10.1016/j.ijheatfluidflow.2017.09.002

10. Jorgensen F.E., 2002, How to Measure Turbulence with Hot-Wire Anemometers - a Practical Guide, Dantec Dynamics A/S, P.O. Box 121, Tonsbakken 16-18, DK-2740 Skovlunde, Denmark

11. Kendall A., Koochesfahani M., 2007, A method for estimating wall friction in turbulent wallbounded flows, Experiments in Fluids, 44, 5, 773-780

12. Knopp T., Schanz D., Schröder A., Dumitra M., Cierpka C., Hain R., Kähler C.J., 2013, Experimental investigation of the log-law for an adverse pressure gradient turbulent boundary layer flow at $\operatorname{Re}_{\theta}=10000$, Flow, Turbulence and Combustion, 92, 1/2, 451-471 
13. Madad R., Harun Z., Chauhan K., Monty J.P., Marusic I., 2010, Skin friction measurement in zero and adverse pressure gradient boundary layers using oil film interferometry, 17th Australasian Fluid Mechanics Conference 2010

14. Mathis R., Hutchins N., Marusic I., 2009, Large-scale amplitude modulation of the small-scale structures in turbulent boundary layers, Journal of Fluid Mechanics, 628 (2009), 311

15. Monty J.P.P., Harun Z., Marusic I., 2011, A parametric study of adverse pressure gradient turbulent boundary layers, International Journal of Heat and Fluid Flow, 32, 3, 575-585

16. Nagib H., Christophorou C., Ruedi J.-D., Monkewitz P., Osterlund J., Gravante S., Chauhan K., Pelivan I., 2004, Can we ever rely on results from wall-bounded turbulent flows without direct measurements of wall shear stress?, 24th AIAA Aerodynamic Measurement Technology and Ground Testing Conference

17. Pailhas G., Barricau P., Touvet Y., Perret L., 2009, Friction measurement in zero and adverse pressure gradient boundary layer using oil droplet interferometric method, Experiments in Fluids, 47, 2, 195-207

18. Segalini A., Rüedi J.-D., Monkewitz P.A., 2015, Systematic errors of skin-friction measurements by oil-film interferometry, Journal of Fluid Mechanics, 773, 298-326

19. Simpson R.L., 1989, Turbulent boundary-layer separation, Annual Review of Fluid Mechanics, 21, 205-234

20. Tanner L.H., Blows L.G., 1976, A study of the motion of oil films on surfaces in air flow, with application to the measurement of skin friction, Journal of Physics E, 9, 3, 194-202

21. Vinuesa R., NagiB H.M., 2015, Enhancing the accuracy of measurement techniques in high Reynolds number turbulent boundary layers for more representative comparison to their canonical representations, European Journal of Mechanics - B/Fluids, November

22. Zanoun E.S., Durst F., NAGiB H., 2003, Evaluating the law of the wall in two-dimensional fully developed turbulent channel flows, Physics of Fluids, 15, 10, 3079-3089 